\title{
CHEOPS SOLAR CELL ASSEMBLIES LIFE TEST
}

\author{
Emilio Fernández Lisbona $^{(1)}$, Gianfelice D’Accolti ${ }^{(1)}$, Joel Asquier ${ }^{(1)}$, Robert Holloway $^{(1)}$, Bob Witteveen $^{(1)}$ \\ Pietro Zanella ${ }^{(2)}$, Luigi Ferrante ${ }^{(2)}$ \\ (1) ESA-ESTEC-Postbus 299-NL 2200 AG Noordwijk-The Netherlands, emilio.fernandez.lisbona@esa.int, \\ Gianfelice.Daccolti@esa.int, Joel.Asquier@esa.int,Robert.Holloway@esa.int,Bob.Witteveen@esa.int \\ ${ }^{(2)}$ Leonardo Finmeccanica, Viale Europa, 20014 Nerviano (MI) - Italy, Pietro.Zanella@leonardocompany.com, \\ Luigi.Ferrante@leonardocompany.com
}

\begin{abstract}
Life tests are performed to determine the stability of solar cell assemblies under worst case operation conditions for long duration. This test is part of the qualification programme to which solar cell assemblies are submitted according to ECSS standards. This paper presents the test set-up configuration (recently implemented in the European Space Power Laboratory), test conditions and test results obtained of the solar cell assembly life test performed under the ESA CHEOPS satellite programme.
\end{abstract}

\section{INTRODUCTION}

\subsection{The CHEOPS Mission}

The CHEOPS (CHaracterizing ExoPlanet Satellite) mission is dedicated to searching for planet transits by means of ultrahigh precision photometry on bright stars already known to host planets. The mission was selected by the Science Programme Committee in November 2012 as the first small mission in the ESA Science Programme. The project passed the system CDR in May 2016 and is targeting a shared launch opportunity in 2018.

To cope with the very challenging schedule and budget, the design and implementation of CHEOPS is articulated around the re-use of an existing, "off-theshelf" platform, the AS250 product line from Airbus DS with some minor adaptations. The aim of the modifications is to meet the power requirements of the mission (battery and solar array resizing) and to provide a cost efficient solution where a downsizing of elements is possible while satisfying the performances. The manufacture of the platform and the integration \& testing of the satellite (including the integration of the scientific instrumentation) is carried out by AIRBUS DS Spain.

The satellite (Fig. 1) is composed of a prismatic body platform, an optical instrument (a photometer developed by a consortium led by the University of Berne) mounted on top of the platform, and three body- mounted solar array panels. The three body-mounted solar array panels have a 67 degree deviation between them, in order to achieve the best illumination in any mission attitude. See [1] for more information about CHEOPS mission.

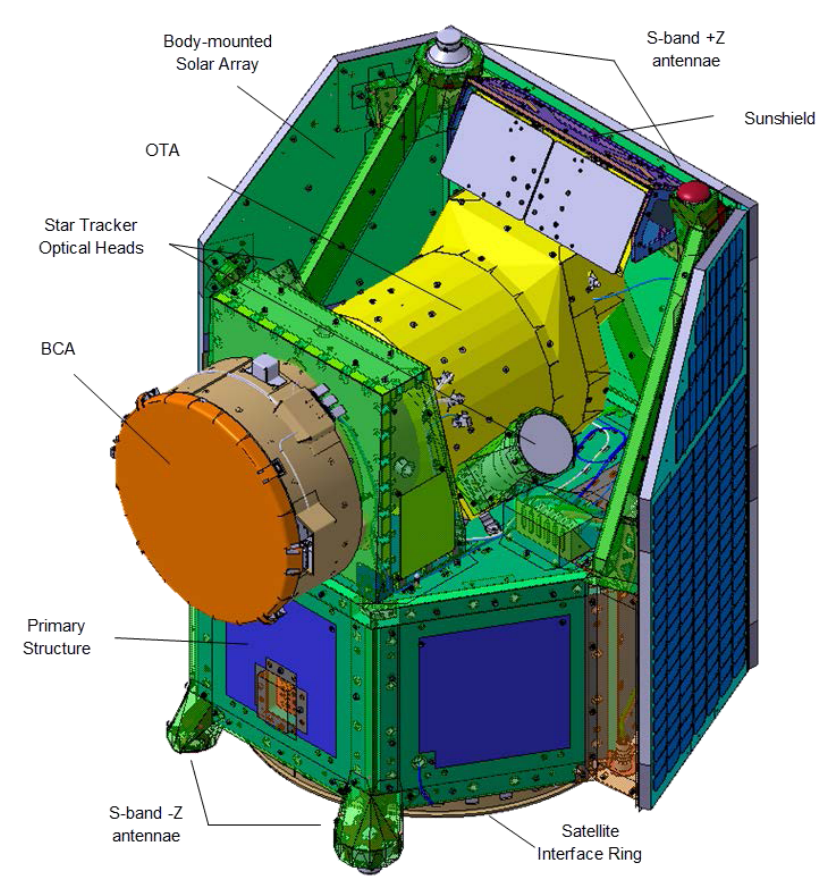

Figure 1. CHEOPS Satellite.

To account for the specific requirements of the CHEOPS mission in terms of sky visibility and straylight rejection (e.g. minimizing Earth stray light) and the mission constraints (e.g. shared launch scenario), a dawn-dusk circular SSO (LTAN 6 a.m. or 6 p.m., and altitude from 650 to $800 \mathrm{~km}$ ) was selected as the nominal orbit. Due to the attitude range and characteristics (e.g. roll around the line-of-sight to maintain the instrument's radiators pointed to cold space), the solar cells are subject to a large number of thermal cycles and elevated cell temperatures, specifically for the central panel, which has resulted in the need for delta-qualification tests of the solar cell assemblies. 


\subsection{Delta-Qualification Tests}

The solar cell assembly delta-Qualification test for CHEOPS mission consisted of the performance of a life test in accordance with ECSS [2] and the specific mission requirements.

\section{LIFE TEST DEFINITION}

The solar cell assembly life test is specified to be performed in a vacuum (less than $2 \times 10^{-5} \mathrm{mBar}$ ) and under 1 solar constant AM0 illuminated simulated conditions.

The duration of the life test is calculated to be considerably less than the real duration of the mission and follows the Arrhenius law, taking into account the total duration of the CHEOPS mission (3.5 years), solar cell activation energy $(0.7 \mathrm{eV}$, being a rather conservative value and in accordance with [2]) and the worst case solar array operational temperature during the mission (i.e. in the range between $120^{\circ} \mathrm{C}$ and $130^{\circ} \mathrm{C}$ ). Therefore, CHEOPS mission conditions require a life test exposure much larger than for GEO and LEO missions and it has been calculated to be 6990 hours at $160^{\circ} \mathrm{C}$.

The life test is split in 3 phases, where the solar cells are exposed at three different temperatures. Therefore, the duration of each phase is calculated to be 2000 hours at $140^{\circ} \mathrm{C}, 2000$ hours at $160^{\circ} \mathrm{C}$ and 2078 hours at $175^{\circ} \mathrm{C}$. The solar cells are loaded close to the maximum power point for $93 \%$ of the time of each phase. During the remaining $7 \%$, the solar cells are biased in reverse voltage to simulate the worst case condition of solar cells current matching in the string with a $0.05 \mathrm{~V}$ margin.

\section{CHEOPS LIFE TEST SAMPLE}

Leonardo-Finmeccanica provided the test sample plate (see Fig.2) consisting of 12 flight representative solar cell assemblies, that were previously qualified for GEO missions under ESA's MTG programme.

The substrate is an stainless steel plate of $300 \times 300$ $\mathrm{mm}^{2}$. The use of stainless steel material helps to avoid cell cracking during vacuum and temperature exposure and provides a reasonable thermal conductivity.

The Solar cell assembly is comprised of 12 AzurSpace 3G30 Triple Junction solar cells with CMG $100 \mu \mathrm{m}$ coverglass with AR coating and Ag plated Invar interconnectors. These are high grade solar cells. The solar cell shunt diode is an AzurSpace SIBD type, but they are not connected in parallel to the cell.

The bus bars are InvarAg single strips and the solar cell laydown adhesive and bus bar potting is RTV-S691.

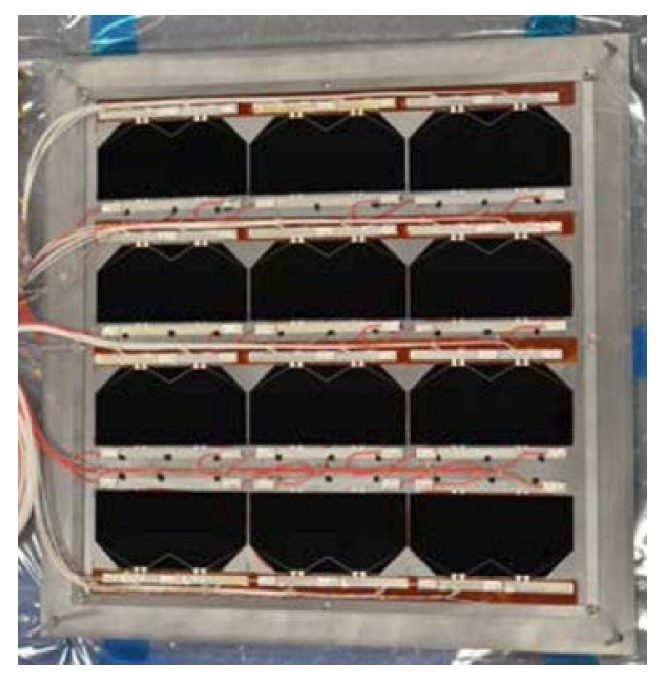

Figure 2. CHEOPS life test sample.

The bus bar bonding patterns and adhesive is CV-21142 and the wire type is 3901-020 ESCC specification.

The plate is equipped with 6 thermocouples of type T bonded below 6 different solar cells.

The 4xPolymide heaters for active thermal control and attached with RTV-S691used for the MTG life test, were removed for the CHEOPS life test.

\section{ESTEC LIFE TEST FACILITY}

The test facility is located in the Solar Generator Laboratory of the Power Systems Division (TEC-EP) at ESTEC and is comprised of:

\subsection{Thermal vacuum facility}

The Vacuum Solar Cell Illumination Facility (VSCIF) is equipped with a quartz glass window that allows AM0 illumination of the solar cells samples. The VSCIF facility is equipped with a XDS10 scroll pump and a BOC Edwards STP-301/451 Series turbomolecular pump and the vacuum level is typically lower than $10^{-6}$ mbar. The VSCIF facility is equipped with a hot plate that uses flat Mica based heaters and allows exposure at constant temperature of the solar cell samples between $120^{\circ} \mathrm{C}$ and $400^{\circ} \mathrm{C}$. No cold trap is used for the CHEOPS life test as the VSCIF facility walls are at much lower temperature $\left(40-60^{\circ} \mathrm{C}\right)$ than the solar cells.

Calcium fluoride windows, placed in the chamber cold locations and in close proximity to the test samples, are used to monitor the deposition on these surfaces of organic contamination during the test. 


\subsection{Solar Simulator}

The solar simulator (SESO SSMD $1 \times 2,5-0-3,5$ ) is a single source system with a Xenon arc lamp of $2500 \mathrm{~W}$. The light beam is composed by reflection on 8 internally mounted mirrors. The SESO solar simulator is placed outside the vacuum facility. The vertical light beam illuminates the solar cell sample through the VSCIF facility window. The illuminated plane has a diameter of $350 \mathrm{~mm}$ at a distance of $650 \mathrm{~mm}$ from the exit window of the simulator.

The solar simulator performance is as follows:

- Intensity: 0.7 solar constant (SC) to $1.7 \mathrm{SC}$.

- Uniformity: $2.0 \%$ at $1 \mathrm{SC}$ in the $350 \mathrm{~mm}$ beam target diameter.

- Spectrum: AM0 at 1 SC, Class A [2].

- Long term stability: $0.2 \%$ over $24 \mathrm{~h}$ at 1 solar constant (SC).

\subsection{Solar cells biasing electrical device}

Solar cells under test are electrically connected to an electric switch box to allow operation in loaded mode and in reverse mode. Each cell is looped with two other solar cells of the same row by means of the open positive terminal. Two groups of 6 cells in parallel are connected to two different power supplies for working in reverse mode. Each solar cells are loaded with a resistor $(2 \Omega)$ when working in loaded mode.

\subsection{Data Acquisition Unit}

The current equivalent and voltage are measured for each solar cell independently during the complete test. The DACQ also records the solar cell temperatures and the equivalent current of two silicon solar cells (one filtered to be sensitive to UV spectral range) in order to control the solar simulator light intensity that degrades due to lamp ageing.

\section{TEST SET-UP VERIFICATION}

\subsection{Test set-up spike verification}

The solar cell electrical circuit was verified to be free of electrical transients for planned (transitions between safe mode and forward/reverse modes) and unexpected events that may occur during the life test execution. A dummy cell was used to verify the transients in the electrical circuit. The dummy cell terminals were directly connected to the measurement oscilloscope. The verification helped to refine the test operational procedure and to prevent transients during nominal test operations. Furthermore an oscilloscope has been connected to one of the cells, in order to monitor unexpected transients, during the complete life test.

\subsection{Solar Simulator Calibration}

The uniformity was measured with the CHEOPS test solar cells that were previously electrically characterized with a calibrated pulsed solar simulator. The overall uniformity is verified to be in the range of \pm $2.0 \%$.

The solar simulator intensity at the solar cell sample test plane is measured with a set of representative component solar cells in the centre of the beam and in the same plane of the CHEOPS test solar cells. In order to characterize the lamp spectral ageing, the light beam intensity is measured at the beginning and at the end of each phase or lamp change. The nominal solar simulator lamp life is around 2000 hours.

\section{LIFE TEST EXECUTION}

The following figures show the temperature, current and voltage of the solar cells monitored during part of phase 2, as an example of the execution of the CHEOPS life test.

Fig. 3 shows the solar cells temperature in loaded mode and in reverse mode. The temperature increase corresponds to the reverse mode step.

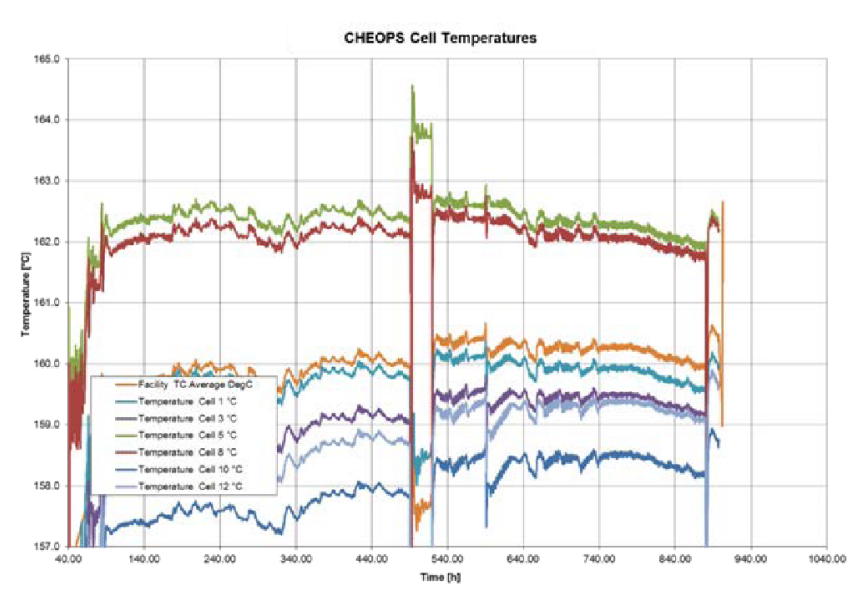

Figure 3. Solar cells temperatures.

Fig. 4 shows the solar cells currents in loaded mode and for a short period in reverse mode. The solar simulator light intensity was increased at $600 \mathrm{~h}$ to compensate for the aging of the lamp. 


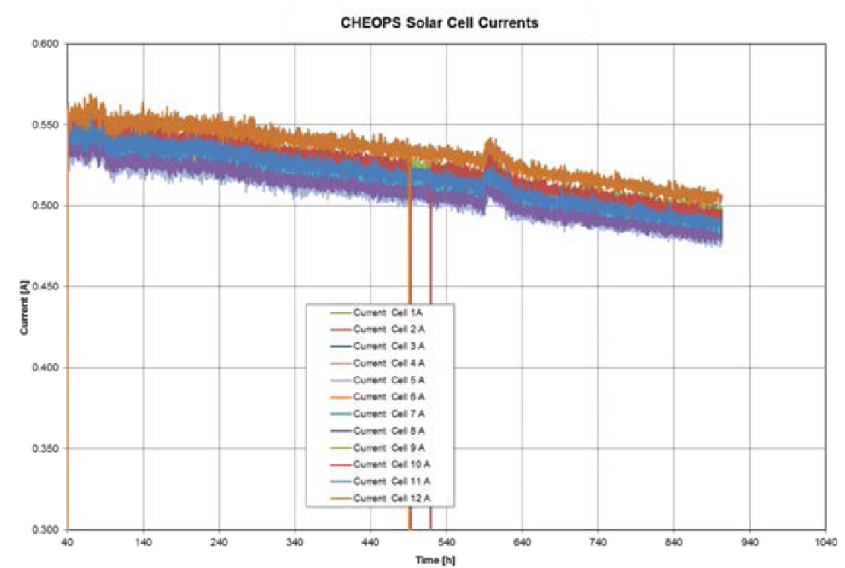

Figure 4. Solar cells currents.

Fig. 5 shows the solar cells voltage in loaded mode and few hours in reverse mode.

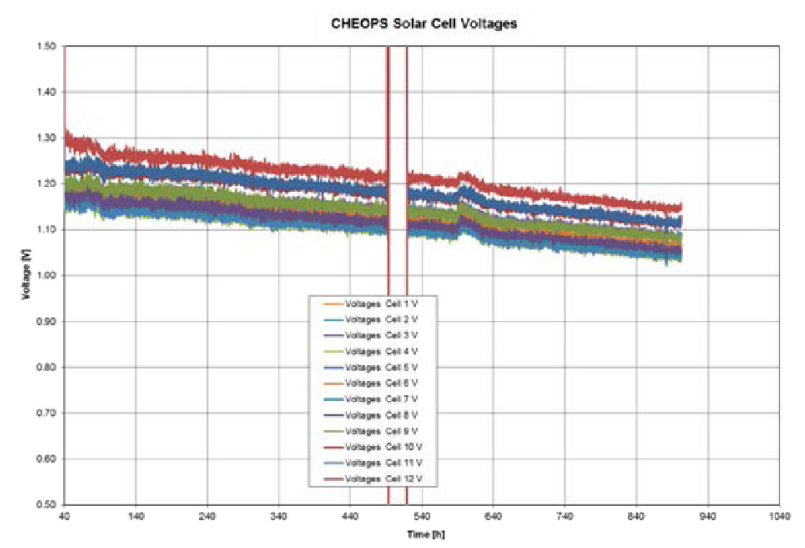

Figure 5. Solar cells voltages.

\section{LIFE TEST RESULTS}

The solar cells are inspected and electrically characterised before the exposure test starts and at the end of each phase. The electrical performance of the solar cell assemblies is measured, at 1 solar constant conditions and at $25^{\circ} \mathrm{C}$. Visual inspection, including electroluminescence inspection of the solar cells, is also carried out. The contamination content of the calcium fluoride windows is measured using IR spectrometry analysis.

After the completion of phases 1 and 2 and more than 1800 hours of exposure at $175^{\circ} \mathrm{C}$, no degradation of solar cell assembly's electrical performance has been recorded and no defects have been identified. The test is planned to be completed towards the end of September 2016.

\section{LESSONS LEARNED}

The Solar simulator optics are sensitive to contamination that becomes fixed to their surfaces as a result of the exposure time, this is particularly due to Ultra Violet (UV) radiation. This contamination decreases the performance of the solar simulator in the UV region and requires the cleaning of the optic surfaces in order to restore it. The cleaning is performed with solvent agents that do not damage the optic coatings.

The process to set-up the reverse bias condition on the solar cells is time consuming because of inter-cell interaction due to the electric switch box design. The solution to implement individual power supplies to bias each solar cell, is seen as a much efficient, even if more expensive.

The solar simulators lamp life may be linked to specific lamp manufacturing batches. Therefore, the implementation of an individual lamp life control has been stablished.

The use of reference component solar cells and monitor, silicon and silicon VI \& IR filtered, solar cells to calibrate and monitor the solar simulator intensity, helped to verify the CHEOPS solar cells performance, together with their measured current at test temperature, after unforeseen test interruptions.

\section{ACKNOWLEDGEMENTS}

The authors are grateful to Airbus DS Spain, in particular to Natalia Perez and Ignacio Diaz, for their work in the definition of the test duration in accordance to the CHEOPS mission requirements. Furthermore the authors are grateful to Natascha Klein and Narimane Brahimi from ESTEC TEC-EPB section for their support in the test set-up preparation and the life test execution.

\section{REFERENCES}

1. Corral van Damme C., Rando N., Asquier N., Ratti F., Southworth R. \& Isaak R. (2016). CHEOPS: ESA First small science mission From mission concept to CDR in 3.5 years. Small Satellites Systems and Services Symposium, 30 May - 3 June 2016, Valletta, Malta.

2. European Cooperation for Space Standardization, ECSS-E-ST-20-08C Rev. 1 "Photovoltaic assemblies and components". 